\section{Use of topical lithium succinate for seborrhoeic dermatitis}

In a small screening study topical lithium succinate ointment was observed to produce a striking improvement in several patients with seborrhoeic dermatitis of the face. We therefore performed a double blind controlled study of this effect.

As pityrosporum yeasts can cause seborrhoeic dermatitis ${ }^{1}$ and lithium inhibits the growth of some fungi ${ }^{2}$ we also tested the inhibitory effect of lithium succinate on the growth of yeasts.

\section{Patients and methods}

We studied 11 men and eight women (aged 15-64) who had all had classical seborrhoeic dermatitis affecting the face for at least three months. Patients were randomly allocated to active or placebo treatment for four weeks, followed by the alternative treatment for a further four weeks. They were instructed to apply the treatment. Although the seborrhoeic dermatitis relapsed at various intervals after active treatment was stopped, it responded to reintroduction of treatment. Lithium succinate did not inhibit the in vitro growth of pityrosporum or the other organisms studied.

\section{Discussion}

The cause of seborrhoeic dermatitis is uncertain, but yeast infection may be aetiologically important. ${ }^{1}$ Topical lithium succinate seems to improve facial seborrhoeic dermatitis strikingly but does not inhibit the growth of pityrosporum yeasts. Presumably it acts as an anti-inflammatory agent by some other mechanism.

Systemic lithium treatment is known to produce a wide range of cutaneous side effects, including acneiform eruptions and exacerbation of psoriasis. Our observation that topical lithium improves seborrhoeic dermatitis whereas systemic lithium exacerbates psoriasis is interesting in that seborrhoeic dermatitis histologically resembles psoriasis, but pustulation, even a a microscopic level, is not a feature of seborrhoeic dermatitis, whereas epidermal invasion by leucocytes is a regular feature of psoriasis.

Mean (SD) effect of topical lithium and placebo in seborrhoeic dermatitis. (Figures are grades on analogue scale 0-10)

\begin{tabular}{|c|c|c|c|c|c|}
\hline & Redness & Scale & Area & Itch & Overall \\
\hline \multicolumn{6}{|c|}{ Patient's assessment } \\
\hline Baseline score & $3 \cdot 7(2 \cdot 0)$ & $3 \cdot 4(2 \cdot 2)$ & $4 \cdot 4(2 \cdot 4)$ & $3 \cdot 4(2 \cdot 4)$ & $4 \cdot 5(1.9)$ \\
\hline Lithium score & $1 \cdot 3(1 \cdot 5)$ & $1 \cdot 1(0 \cdot 8)$ & $1.8(1.9)$ & $1.5(1.5)$ & $1 \cdot 7(1 \cdot 7)$ \\
\hline Placebo score & $3 \cdot 5(2 \cdot 3)$ & $2 \cdot 5(1 \cdot 9)$ & $3 \cdot 5(2 \cdot 4)$ & $2 \cdot 8(2 \cdot 2)$ & $3 \cdot 7(2 \cdot 2)$ \\
\hline \multicolumn{6}{|c|}{ Lithium $v$ baseline: } \\
\hline$t$ Value & $5 \cdot 0$ & $4 \cdot 0$ & $4 \cdot 8$ & $2 \cdot 9$ & $6 \cdot 1$ \\
\hline p Value & $<0.001$ & $<0.001$ & $<0.001$ & $<0.02$ & $<0.001$ \\
\hline \multicolumn{6}{|c|}{ Placebo $v$ baseline: } \\
\hline$t$ Value & 0.4 & $1 \cdot 5$ & $2 \cdot 1$ & 0.9 & $1 \cdot 7$ \\
\hline p Value & NS & NS & NS & NS & NS \\
\hline \multicolumn{6}{|c|}{ Lithium $v$ placebo: } \\
\hline$t$ Value & $4 \cdot 2$ & $3 \cdot 7$ & $3 \cdot 7$ & $2 \cdot 2$ & 3.9 \\
\hline p Value & $<0.001$ & $<0.001$ & $<0 \cdot 001$ & $<0.05$ & $<0.001$ \\
\hline \multicolumn{6}{|c|}{ Doctor's assessment } \\
\hline Baseline score & $4 \cdot 4(2 \cdot 1)$ & $4 \cdot 3(2 \cdot 4)$ & $4 \cdot 8(1 \cdot 9)$ & & $4 \cdot 5(1 \cdot 7)$ \\
\hline Lithium score & $1.0(1.4)$ & $1 \cdot 0(1 \cdot 3)$ & $1 \cdot 0(1 \cdot 1)$ & & $1 \cdot 1(1 \cdot 3)$ \\
\hline Placebo score & $3 \cdot 4(1 \cdot 8)$ & $2.9(1.9)$ & $3 \cdot 3(2 \cdot 0)$ & & $3 \cdot 2(1 \cdot 8)$ \\
\hline \multicolumn{6}{|c|}{ Lithium $v$ baseline: } \\
\hline$t$ Value & 6.8 & $4 \cdot 6$ & $6 \cdot 3$ & & $5 \cdot 7$ \\
\hline $\mathrm{p}$ Value & $<0.001$ & $<0.001$ & $<0.001$ & & $<0.001$ \\
\hline \multicolumn{6}{|c|}{ Placebo $v$ baseline: } \\
\hline$t$ Value & $1 \cdot 5$ & $2 \cdot 2$ & $2 \cdot 3$ & & $2 \cdot 2$ \\
\hline p Value & NS & $<0.05$ & $<0.05$ & & $<0.05$ \\
\hline \multicolumn{6}{|c|}{ Lithium $v$ placebo: } \\
\hline$t$ Value & $5 \cdot 6$ & $4 \cdot 5$ & $4 \cdot 6$ & & $5 \cdot 4$ \\
\hline $\mathrm{p}$ Value & $<0.001$ & $<0.001$ & $<0.001$ & & $<0.001$ \\
\hline
\end{tabular}

ointment sparingly to the affected area of skin twice daily. No other topical preparation was used during the study. Fourteen of the patients had used a mild topical steroid preparation before the study: seven of these used the placebo first and seven used the active preparation first. The lithium ointment contained lithium succinate $8 \%$, zinc sulphate $0.05 \%$, and $d l$ - $\alpha$ tocopherol in a lanolin base.

The placebo contained lanolin base only.

The severity of seborrhoeic dermatitis was graded by the patient and the same doctor at zero, four, and eight weeks. A separate assessment was made of erythema, scale, the area affected, and the overall impression of the severity using separate linear analogue scales. The patients also assessed itching. The values obtained after four weeks' treatment were compared with the baseline values (week 0 ), and the results of active and placebo treatment were also compared with each other using paired $t$ tests.

The possible inhibitory effect of lithium succinate on the growth of pityrosporum yeasts and other fungi was studied using standard methods. ${ }^{3}$ The minimal inhibitory concentration was measured against pityrosporum yeasts grown on diagnostic sensitivity test medium (Oxoid), with glyceryl monostearate $(2.5 \mathrm{~g} / \mathrm{l})$ and Tween $80(2 \mathrm{ml} / \mathrm{l})$. Lithium succinate was diluted in water, giving concentrations in the plates of $1-150 \mathrm{mg} / \mathrm{l}$. Five isolates of Pityrosporum orbiculare and two of $P$ ovale were tested. The minimal inhibitory concentration of lithium against Candida albicans, Trichophyton rubrum, and $T$ mentagrophytes was also studied.

\section{Results}

Two of the 19 patients withdrew from the study: one found the active treatment effective and did not wish to change to placebo, the other found that both treatments irritated her skin. Of the remaining 17 patients, 14 showed improvement with lithium ointment, two had no response to either treatment, and one responded to placebo. All assessments by doctor and patients showed a significant improvement in the mean score after four weeks when lithium was compared with the baseline; lithium was also significantly more effective than placebo (table).

The response to topical lithium generally occurred within the first two weeks of
Dr David Horrobin of Scotia Pharmaceuticals, PO Box 818, Kentville, Nova Scotia $\mathrm{B} 4 \mathrm{~N} 4 \mathrm{H} 8$, Canada, suggested this study and provided the active and placebo ointments.

1 Ford GP, Farr PM, Ive FA, Shuster S. The response of seborrhoeic dermatitis to ketoconazole. $\mathrm{Br} f$ Dermatol 1984;iii:603-7.

2 Kurita $N$, Funabashi $M$. Growth inhibitory effect on fungi of alkali cations and monovalen inorganic anions and antagonism among different alkali cations. Agricultural and Biological Chemistry 1984;48:887-94.

3 Faergemann J, Fredriksson T. The antimycotic activity in vitro of five diols. Sabouradia 1980;18:287-93.

4 Heng MCY. Cutaneous manifestations of lithium toxicity. Br f Dermatol 1982;106: 107-9.

5 Lazarus GS, Gilgor RS. Psoriasis, polymorphonuclear leucocytes, and lithium carbonate: an important clue. Arch Dermatol 1979;115:1183-4.

(Accepted 24 September 1985)

Department of Dermatology, Bristol Royal Infirmary, Bristol BS2 8HW

J BOYLE, MRCP, senior registrar

J L BURTON, FRCP, consultant

Department of Dermatology, Central Hospital, Vasteras, Sweden

J FAERGEMANN, MD, consultant

Correspondence to: Dr Burton.

\section{Correction}

Olsalazine in active ulcerative colitis

An error occurred in this paper by Dr W S Selby et al. The description of the enema trial stated that the active enema contained $2 \mathrm{~g}$ olsalazine; this should have read $1 \mathrm{~g}$. 